\title{
Near-Infrared Spectroscopic Examination of Alterations in Brain Surface Blood Flow following Stellate Ganglion Block
}

\author{
Fukami Nakajima ${ }^{1,2,3 *}$, Mariko Kawate ${ }^{1}$, Koshi Makita ${ }^{2}$ \\ ${ }^{1}$ Nishiogi Pain Clinic, Tokyo, Japan \\ ${ }^{2}$ Department of Anesthesiology, Graduate School of Medical and Dental Sciences, Tokyo Medical and Dental University, Bunkyo-ku, Tokyo, Japan \\ ${ }^{3}$ Department of Locomotor Science, Institute of Medical Science, Tokyo Medical University, Shinjuku-ku, Tokyo, Japan
}

Received: August 25, 2016; Accepted: September 06, 2016, Published: September 09, 2016

*Corresponding author: Fukami Nakajima, Department of Anesthesiology, Graduate School of Medical and Dental Sciences, Tokyo Medical and Dental University, 1-5-45 Yushima, Bunkyo-ku, Tokyo 113-8519, Japan, Tel: +81-3-3345-0165; Fax: +81-3-6302-0265; E-mail: nakmane@tmd.ac.jp

\begin{abstract}
Background: Stellate Ganglion Block (SGB) is used to treat pain and other disorders, but the target diseases of SGB are controversial because the mechanisms underlying SGB remain unclear. Increased cerebral blood flow is one of the processes presumed to be involved. The aim of this study was to clarify the effects of SGB by using nearinfrared spectroscopy to measure brain surface blood flow during and after SGB.

Methods: The participants were nine healthy staff volunteers. Near-infrared spectroscopy was applied to subjects' heads, and the same operator performed a right SGB (1\% mepivacaine $4 \mathrm{ml})$. Changes in oxy-hemoglobin (oxy-Hb) concentration were measured from approximately $30 \mathrm{~s}$ before administering SGB until approximately after $30 \mathrm{~min}$.

Results: For the mean brain waves between the right (block) side channels, oxy-Hb concentration immediately increased after SGB in four subjects, while it temporarily decreased after SGB and then increased in five subjects. For the mean brain waves between the left (contralateral) side channels, oxy-Hb concentration markedly decreased after SGB in four subjects. In three subjects, it temporarily increased immediately after SGB but decreased after approximately $15 \mathrm{~min}$. almost no change in oxy-Hb concentration was observed in the remaining two subjects. Overall, oxy-Hb concentration increased in all subjects on the right (block) side.

Discussion: Increased oxy-Hb concentrations suggest that SGB relaxed vascular tone, increased the capillary bed, and enhanced blood flow. However, oxy-Hb concentration temporarily decreased before it increased in five subjects. We assume that during the temporary decrease, blood flow was unable to keep up with this rate of increase in the capillary bed. In four subjects, oxy-Hb concentration markedly decreased contralateral to the block. This may have been due to higher blood volume on the block side, but the reasons are not fully known.
\end{abstract}

Keywords: Stellate ganglion block; Optical topography; Cerebral blood flow

\section{Introduction}

The stellate ganglion block (SGB) is utilized for face, neck, and upper limb pain [1,2] and treating hot flashes [3]. It is employed to treat systemic diseases such as autonomic imbalance and fibromyalgia in Japan, where it has a broader range of indications than other countries [4-6]. However, target diseases of SGB are sometimes controversial [4] because it is unclear why this therapy works. One of its presumed mechanisms of action is increased cerebral blood flow [7] owing to interruption of the sympathetic nervous system, but this is not fully understood. One study [8] measured cerebral blood flow using single-photon emission computed tomography and assessed tympanic temperature change after SGB. Others measured tympanic temperature [9], skin temperature [10], and regional cerebral oxygen saturation $[11,12]$ to clarify how cerebral blood flow changes after SGB, but we need more detailed information to understand the underlying mechanisms. The brain comprises many unique areas, and it is important to learn how blood flow is altered in different regions and over time after SGB. This will require repeatedly measuring cerebral blood flow with a non-invasive, continuous method to determine which diseases will benefit from SGB. In this study, we used multichannel near-infrared spectroscopy (NIRS) to noninvasively measure hemoglobin concentration changes in the cerebral cortex after SGB.

\section{Methods}

\section{Subjects}

This study was approved by the clinic's ethics committee (Ref no.: NS201401). The study population comprised nine healthy volunteers (eight female, 1 male). All subjects were staff members of the clinic; they all fully understood SGB and the research and provided consent to participate in the study. Tests were conducted to confirm that none of the subjects had a tendency to bleed. All subjects were right-handed.

\section{Experimental methods}

NIRS measures oxy-hemoglobin (oxy-Hb) and deoxyhemoglobin (deoxy-Hb) concentration changes at a depth of 2 to $3 \mathrm{~cm}$ from the brain surface using near infrared light, thereby enabling detection of cerebral blood flow. In April 2009 in Japan, NIRS was approved by the health ministry as an "advanced medical technology" to assist the diagnoses of psychiatric illnesses 
$[13,14]$. Even though there are some controversies about using NIRS for diagnosing psychiatric disease, NIRS is the standard accepted technique of the Japanese health ministry. In this study, we used a 48-channel NIRS (ETG-4000 Hitachi Medical Co. Tokyo, Japan), which enabled continuous non-invasive measurement of cerebral blood flow changes at each location on the brain surface with a resolution of approximately $0.1 \mathrm{~s}$.

Subjects lay supine in a bed with a 48-channel NIRS attached to their head (Figure 1). SGB was performed by injecting $4 \mathrm{ml}$ of $1 \%$ mepivacaine with a $25 \mathrm{G}$ needle into the vicinity of the base of the right transverse process of the 7 th cervical vertebra. This occurred over approximately 20s. The same operator performed the procedure for all subjects. SGB was considered successful based on the appearance of Horner's sign and hemi-facial flushing.

The measurements began approximately 30 s before initiating the procedure, and the oxy- $\mathrm{Hb}$ and deoxy- $\mathrm{Hb}$ concentrations immediately before the block were set as the reference. Changes in cerebral blood flow were calculated by measuring changes in oxy- $\mathrm{Hb}$ and deoxy-Hb concentrations during the block and for 30 min afterwards.

Beginning immediately after the procedure, the subject used their left hand to apply pressure to the puncture site for approximately $10 \mathrm{~min}$ to stop blood flow. We confirmed the extent of hemostasis by removing pressure a number of times halfway through the procedure. Subjects were asked to stay as still as possible during the measurements and remain relaxed without thinking.

\section{Results}

Figure $2 \mathrm{~A}$ and $2 \mathrm{~B}$ shows the mean raw waveforms between channels for each of the left and right hemisphere locations in two subjects. Changes in hemoglobin concentration associated with brain activity are thought to be derived from increased blood flow associated with greater blood volume in cerebral cortex capillary beds. The data measured in 48 channels are represented by brain waves divided into two locations: the mean brain waves for the changes in $\mathrm{Hb}$ in channels close to the right and left sides of the head.

In the subject shown in Figure 2A, the oxy-Hb concentration on the right (block side) temporarily decreased after SGB, then increased. In five of the nine subjects, the oxy-Hb concentration on the right side increased after initially decreasing. In the remaining four subjects, the oxy-Hb concentration on the right side increased immediately after SGB.

The oxy-Hb concentration in the mean brain waves between channels on the left (contralateral) side of the head decreased after temporarily increasing in the subject shown in Figure 2B. This trend of a temporary oxy-Hb concentration increase on the left side followed by a decrease was observed in three subjects. In four subjects, the oxy-Hb concentration on the left side significantly decreased immediately after SGB. In the remaining two subjects, almost no change was seen in the oxy- $\mathrm{Hb}$ concentration on the left side.

\section{Discussion}

In this study, we used NIRS to show that oxy-Hb concentration increased on the block (right) side of the head after SGB in all subjects. This suggests that the treatment relaxes vascular tone, increases the capillary bed, and elevates oxy-Hb concentration due to greater blood flow. However, in five subjects the oxy$\mathrm{Hb}$ concentration temporarily decreased before increasing. We assume that during this period, the capillary bed increased rapidly, with the increase in blood volume unable to keep up. It is likely that the blood volume then increased in response to the increased capillary bed, with a corresponding increase in oxy-Hb concentration. Our results are in accordance with those reported by [11].

Compressive hemostasis was performed for approximately 10 min after SGB, which may have affected cerebral blood flow. Changes in oxy-Hb levels can be more accurately evaluated without pressure to the injection site; however, serious adverse effects including death caused by retropharyngeal hematoma

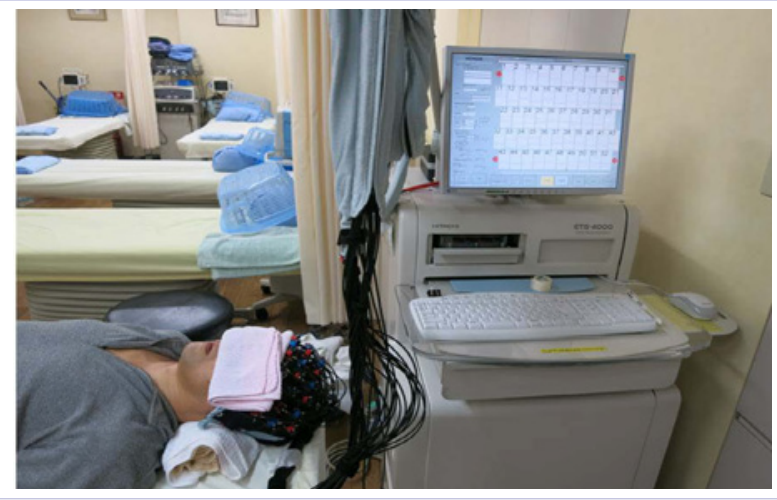

Figure 1: Use of 48-channel NIRS.

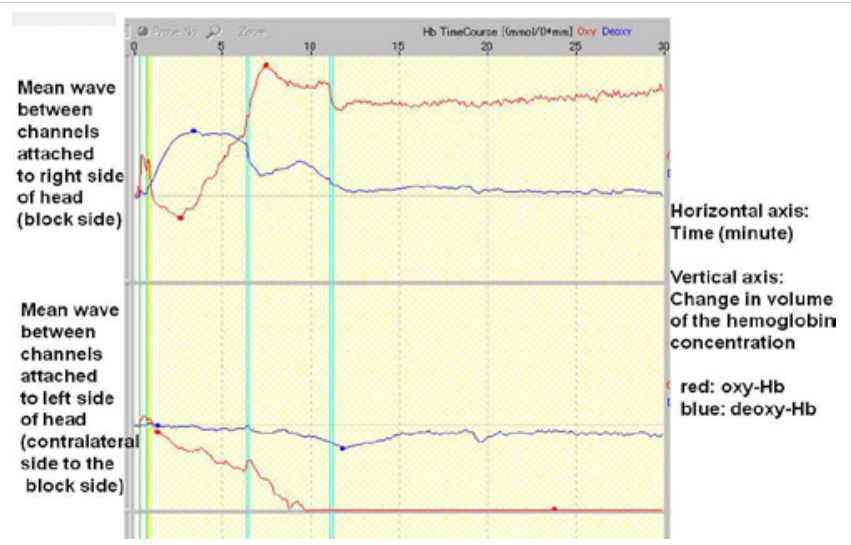

Figure 2a: The mean raw waveforms between channels for the left and right hemispheres.

The horizontal axis shows the time (in minutes) from the start of measurement, with SGB initiated approximately $30 \mathrm{~s}$ later. The space between the two yellow and green lines represents the time the SGB drug solution was injected. The pale blue vertical line indicates the extent of hemostasis as confirmed by temporarily removing the pressure source. The vertical axis represents the change in $\mathrm{Hb}$ concentrations, with the red and blue lines representing oxy-Hb and deoxy-Hb, respectively.. 


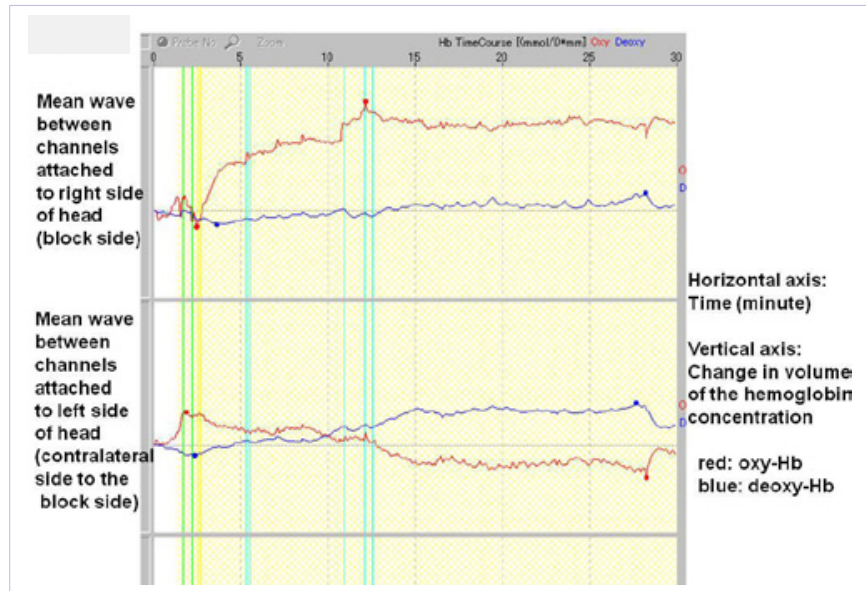

Figure 2b: The horizontal axis shows the time (in minutes) from the start of measurement, with SGB initiated approximately $30 \mathrm{~s}$ later. The space between the two yellow and green lines represents the time the SGB drug solution was injected. The pale blue vertical line indicates the extent of hemostasis as confirmed by temporarily removing the pressure source. The vertical axis represents the change in Hb concentrations, with the red and blue lines representing oxy-Hb and deoxy-Hb, respectively.

have been reported following SGB $[15,16,17]$, so compressive hemostasis is essential. Although the degree of the effect of compression on oxy-Hb levels is unclear, it may have decreased blood flow on the compression side when pressure was applied, which led to lower oxy-Hb levels compared to baseline. If the effect of compression was excluded, oxy-Hb levels may be higher in the blocked side.

In seven subjects, oxy-Hb concentration significantly decreased on the left (contralateral to the block) side. Potential explanations for this phenomenon include blood utilization due to increased blood volume on the block side, decreased blood volume on the contralateral side (Steal phenomenon), and a capillary bed increase on the contralateral side. The blood volume may have been unable to keep up with the rising rate of increase. Another possibility is that drowsiness after SGB reduced the required volume of oxy- $\mathrm{Hb}$. On the block side, the effect of blood volume increase outweighs the effect of drowsiness; therefore, the oxy- $\mathrm{Hb}$ concentration remains elevated on the block side. However, these mechanisms are still not fully understood. In three subjects, the oxy-Hb concentration on the left side of the head decreased after temporarily increasing; the reason for this is also unknown.

Understanding SGB mechanisms is also important for identifying populations that may be harmed by the procedure. The observed decrease on the contralateral side led us to consider that SGB is not indicated in patients with cerebral blood vessel narrowing on the contralateral side.

Further investigation is required to clarify the mechanism of action of SGB.

\section{Conclusion}

In conclusion, oxy-Hb concentrations increased on the block side in all nine subjects, and oxy-Hb concentrations decreased on the contralateral side in seven subjects.

\section{Acknowledgements \\ We thank the staff at Nishiogi Pain Clinic in Tokyo, Japan.}

\section{Declarations}

No funding sources were provided. All authors have contributed equally to this manuscript.

\section{Disclaimers} funder.

This article is not an official position of the institution or

\section{Sources of Support}

We did not receive any support for this study.

\section{Conflict of interest: NA \\ Ethical Approval}

This study was approved by the clinic's ethics committee (NS201401). All participants fully understood the SGB procedure and research and consented to participate.

Written informed consent was obtained from the volunteer subjects for publication of this article. A copy of the written consent form is available for review by the Editor-in-Chief of this journal.

\section{References}

1. Elias M. Cervical sympathetic and stellate ganglion blocks. Pain Physician. 2000;3(3):294-304.

2. Abdi S, Zhou Y, Patel N, Saini B, Nelson J. A new and easy technique to block the stellate ganglion. Pain Physician. 2004;7(3):327-331.

3. van Gastel P, Kallewaard JW, van der Zanden M, de Boer H. Stellateganglion block as a treatment for severe postmenopausal flushing. Climacteric. 2013;16(1):41-47. doi:10.3109/13697137.2012.709889.

4. Uchida K, Tateda T, Hino H. Novel mechanism of action hypothesized for stellate ganglion block related to melatonin. Med Hypotheses. 2002;59(4):446-449. doi:10.1016/S0306-9877(02)00158-5.

5. Nakajima F, Komoda A, Aratani S, Fujita H, Kawate M, Nakatani K, et al. Effects of xenon irradiation of the stellate ganglion region on fibromyalgia. J Phys Ther Sci. 2015;27(1):209-212. doi:10.1589/ jpts.27.209.

6. Nakajima F, Aratani S, Fujita H, Nakatani K, Makita K, Nakajima T. A case of fibromyalgia involving pain throughout the body treated with site-specific targeted pain control. Springerplus. 2016;5(1):1027. doi:10.1186/s40064-016-2572-z.

7. Umeyama T, Kugiyama T, Yoshida S, Wakasugi B, Hanaoka K. The effect of stellate ganglion block on patients with amenorrhea. J Jpn Soc Clin Anesth. 1994;14:109-114

8. Umeyama T, Kugiyama T, Ogawa T, Kandori Y, Ishizuka A, Hanaoka K. Changes in cerebral blood flow estimated after stellate ganglion block by single photon emission computed tomography. J Auton Nerv Syst. 1995;50(3):339-346.

9. Murakawa K, Noma K, Ishida K, Matsuda M, Maeda S, Nishimura M, et al. Changes of tympanic temperature by stellate ganglion block. Masui. 1995;44(6):824-827. 
10. Stevens RA, Stotz A, Kao TC, Powar M, Burgess S, Kleinman B. The relative increase in skin temperature after stellate ganglion block is predictive of a complete sympathectomy of the hand. Reg Anesth Pain Med. 1998;23(3):266-270.

11. Park HM, Kim TW, Choi HG, Yoon KB, Yoon DM. The change in regional cerebral oxygen saturation after stellate ganglion block. Korean J Pain. 2010;23(2):142-146. doi: 10.3344/kjp.2010.23.2.142.

12. Okubo Y, Ogata H. Brain blood volume measured with near infrared spectroscopy increased after stellate ganglion block. Masui. 1995;44(3):423-427.

13. First do no harm. Nature. 2011;469(7329):132. Doi:10.1038/469132a.
14.David Cyranoski. Neuroscience: Thought experiment Nature. 2011;469(7329):148-149. doi:10.1038/469148a.

15. Takanami I, Abiko T, Koizumi S. Life-threatening airway obstruction due to retropharyngeal and cervicomediastinal hematomas following stellate ganglion block. Thorac Cardiovasc Surg. 2009;57(5):311-312. doi:10.1055/s-2008-1038845

16. Higa K, Hirata K, Hirota K, Nitahara K, Shono S. Retropharyngeal hematoma after stellate ganglion block: analysis of 27 patients reported in the literature. Anesthesiology.2006;105(6):1238-1245.

17. Okuda Y, Urabe K, Kitajima T. Retropharyngeal or cervicomediastinal haematomas following stellate ganglion block. Eur J Anaesthesiol. 2003;20(9):757-759. 